\title{
Optimization of Benzothiazole and Thiazole Hydrazones as Inhibitors of Schistosome BCL-2
}

William Nguyen, ${ }^{\dagger}+$, Erinna F. Lee, ${ }^{\perp, \S, ¥,}$ Marco Evangelista, ${ }^{\S}$ Mihwa Lee, ${ }^{\perp}$ Tiffany J. Harris, ${ }^{\S}$ Peter M. Colman, ${ }^{\dagger}{ }^{\dagger}$ Nicholas A. Smith, ${ }^{\perp}$ Luke B. Williams, ${ }^{\dagger}$ Kate E. Jarman, ${ }^{\dagger},{ }^{\dagger}$ Kym N. Lowes, ${ }^{\dagger,}$ Cécile Haeberli, ${ }^{\infty}, @$ Jennifer Keiser, ${ }^{\infty}, @$ Brian J. Smith, ${ }^{\perp}$ W. Douglas Fairlie, ${ }^{\perp, \S, ¥, *}$ and Brad E. Sleebs. ${ }^{\dagger, \hbar}, \epsilon, *$

$\dagger$ The Walter and Eliza Hall Institute of Medical Research, Parkville 3052, Australia.

$\$$ Department of Medical Biology, The University of Melbourne, Parkville 3010, Australia.

${ }^{\perp}$ La Trobe Institute for Molecular Science, La Trobe University, Melbourne 3086, Australia.

$\S$ Olivia Newton-John Cancer Research Institute, Heidelberg 3084, Australia.

$¥$ School of Cancer Medicine, La Trobe University, Melbourne 3086, Australia.

${ }^{€}$ Department of Veterinary Biosciences, The University of Melbourne, Parkville 3010, Australia.

${ }^{\infty}$ Department of Medical Parasitology and Infection Biology, Swiss Tropical and Public Health Institute, Basel, Switzerland.

@ University of Basel, Basel, Switzerland.

* Correspondence addressed to,

Brad Sleebs, sleebs@wehi.edu.au

W. Douglas Fairlie, doug.fairlie@,onjcri.org.au 


\section{Index}

\section{Page}

3 Synthetic pathways for specific hydrazine intermediates (Schemes S1 - S3).

4 Structure of compound $\mathbf{4 3}$ modelled to sBCL2 (Figure S1).

5 sBCL-2 dose response curves for selected compounds (Figure S2).

6 BCL-XL dose response curves for selected compounds (Figure S3).

7 MCL-1 dose response curves for selected compounds (Figure S4).

8 X-ray diffraction data and refinement statistics (Table S1).

9 Evaluation of compounds in a S. mansoni parasite death assay (Table S2).

10 References. 
<smiles>NC(=S)Nc1ccccc1Br</smiles>

$51,83 \%$<smiles></smiles>

$52,12 \%$

$53,63 \%$

Scheme S1. General synthetic pathway to access intermediate 53. Reagents and conditions:

(a) $\mathrm{HCl}$, ammonium thiocyanate, $100^{\circ} \mathrm{C}$; (b) $\mathrm{Br}_{2}, \mathrm{CHCl}_{3}, 20{ }^{\circ} \mathrm{C}$ to $60{ }^{\circ} \mathrm{C}$ then $20^{\circ} \mathrm{C}$; (c) hydrazine hydrate, ethylene glycol, $198^{\circ} \mathrm{C}$.

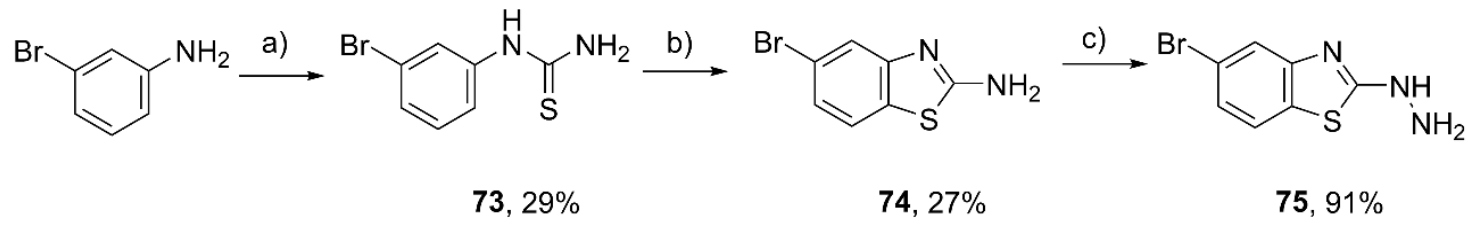

Scheme S2. General synthetic pathway to yield intermediate 75. Reagents and conditions: (a) thiocyanic acid, $\mathrm{NaHSO}_{3}, 20 \% \mathrm{HCl}, 100^{\circ} \mathrm{C}$; (b) $\mathrm{Br}_{2}, \mathrm{CHCl}_{3}, 20^{\circ} \mathrm{C}$ to $60^{\circ} \mathrm{C}$ then $20^{\circ} \mathrm{C}$; (c) hydrazine hydrate, ethylene glycol, $198^{\circ} \mathrm{C}$.<smiles>Nc1c(I)cccc1C(=O)O</smiles><smiles>[R]NC(=O)c1cccc2sc(SC)nc12</smiles>

$$
\begin{aligned}
& \mathrm{R}=\mathrm{CH}_{3} \quad 93,67 \% \\
& =\mathrm{CH} 2 \mathrm{CyPr} \quad 96,67 \% \\
& =\mathrm{Ph} \quad 99,66 \% \\
& =\mathrm{Bzl} \quad 102,95 \% \\
& =\left(\mathrm{CH}_{2}\right)_{2} \mathrm{Ph} \quad \text { 105. 86\% }
\end{aligned}
$$

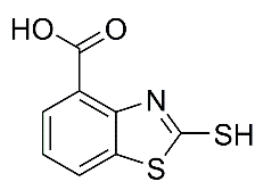

$66,60 \%$<smiles>[R]NC(=O)c1cccc2sc(S(C)(=O)=O)nc12</smiles>

$94,96 \%$

97, $100 \%$

$100,79 \%$

$103,61 \%$

$106,81 \%$ b)<smiles>CSc1nc2c(C(=O)O)cccc2s1</smiles>

$67,53 \%$

Scheme S3. General synthetic pathway to generate substituted 2-hydrazinobenzothiazoles 95, 98, 101, 104 and 107. Reagents and conditions: (a) potassium ethyl xanthogenate, DMF, 120 ${ }^{\circ} \mathrm{C}$ (b) MeI, TEA, DCM, $20{ }^{\circ} \mathrm{C}$; (c) $\mathrm{HBTU}, \mathrm{R}-\mathrm{NH}_{2}$, DMF; (d) $\mathrm{KMnO}_{4}, \mathrm{AcOH}, \mathrm{H}_{2} \mathrm{O}, 20{ }^{\circ} \mathrm{C}$; (e) hydrazine hydrate, EtOH, $90{ }^{\circ} \mathrm{C}$. 


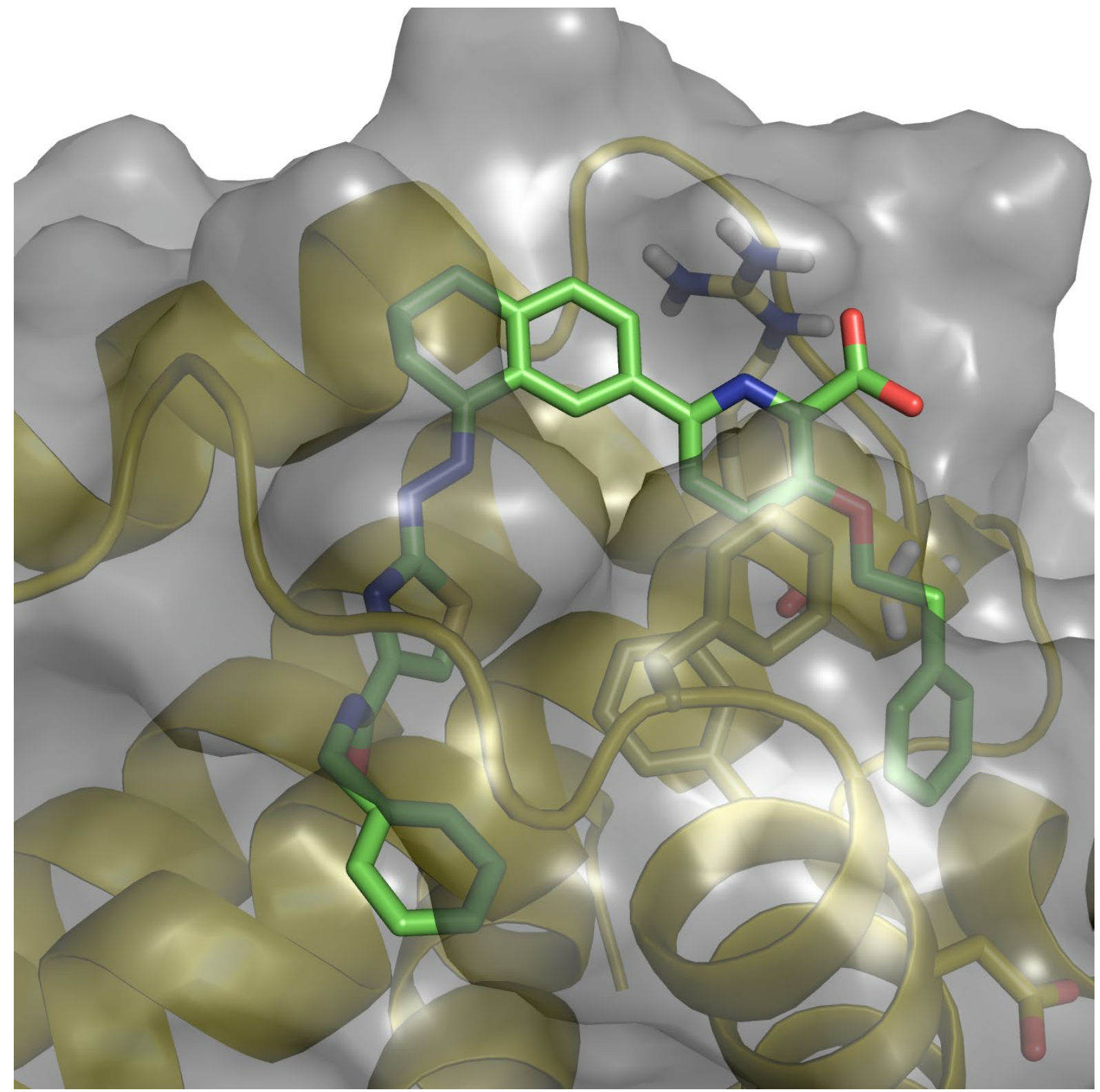

Figure S1. Compound 43 modelled (green) modelled in complex with sBCL-2 (gold) showing the $N$-benzyl thiazole carboxamide moiety extending out of the open $\mathrm{P} 2$ cavity. 

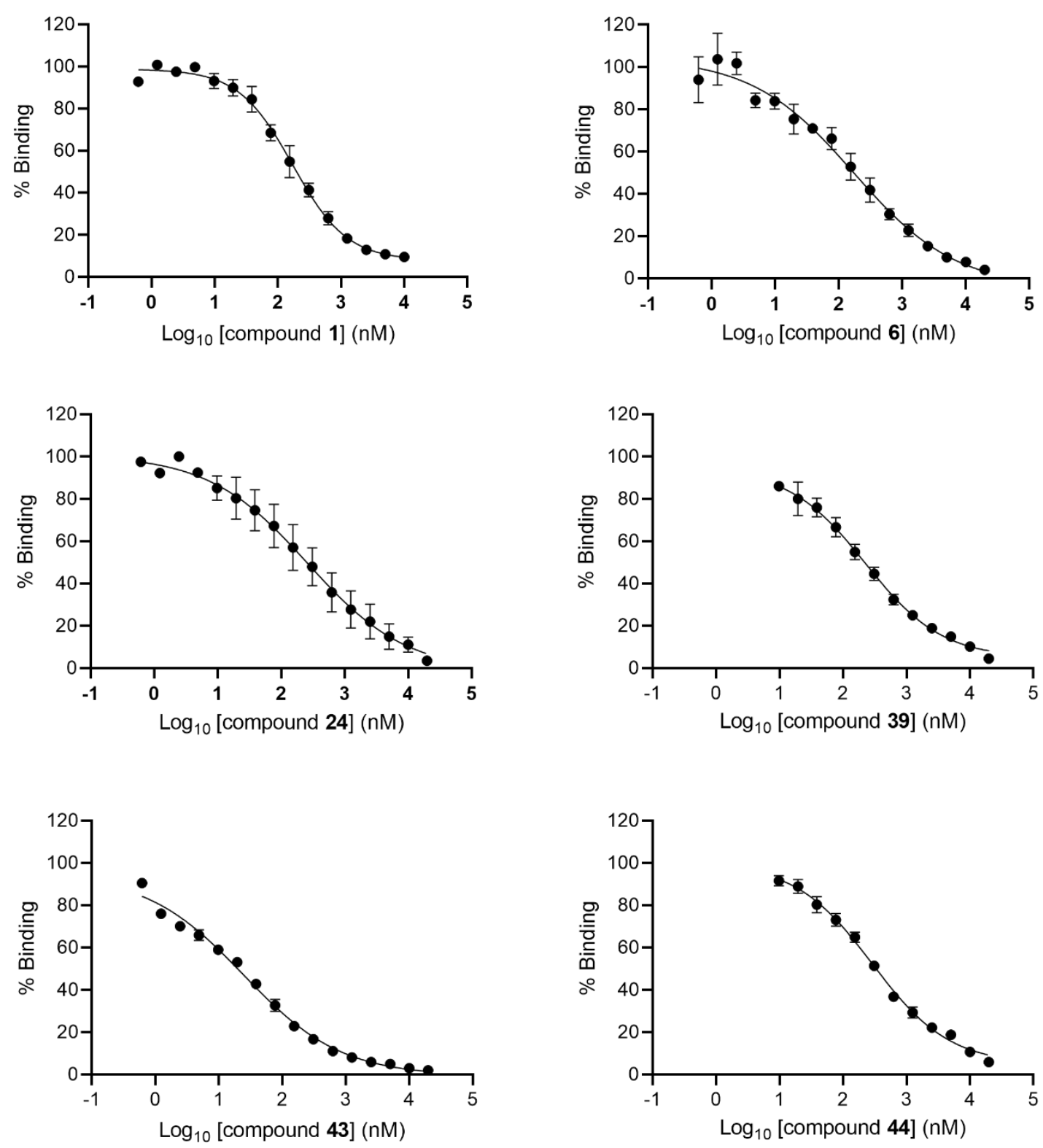

Figure S2. Dose response curves of representative compounds against sBCL-2. IC50 data represents means and SDs for three independent experiments on compounds in a 10-point dilution series against sBCL-2 using an AlphaScreen assay format. 

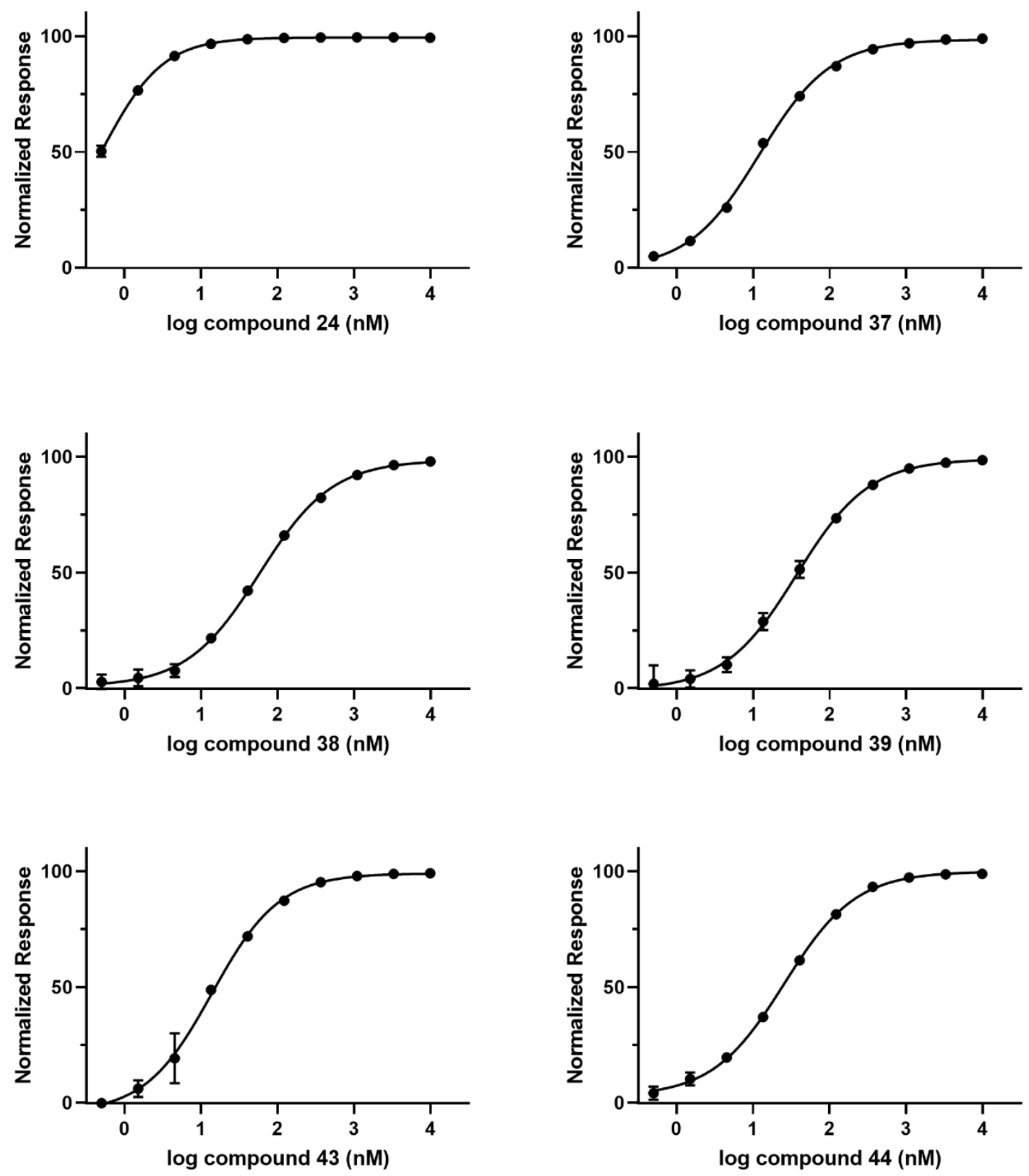

Figure S3. Dose response curves of representative compounds against BCL-XL. IC50 data represents means and SDs for four technical experiments on compounds in a 10-point dilution series against BCL-XL using the AlphaScreen assay format., ${ }^{1,2}$ 

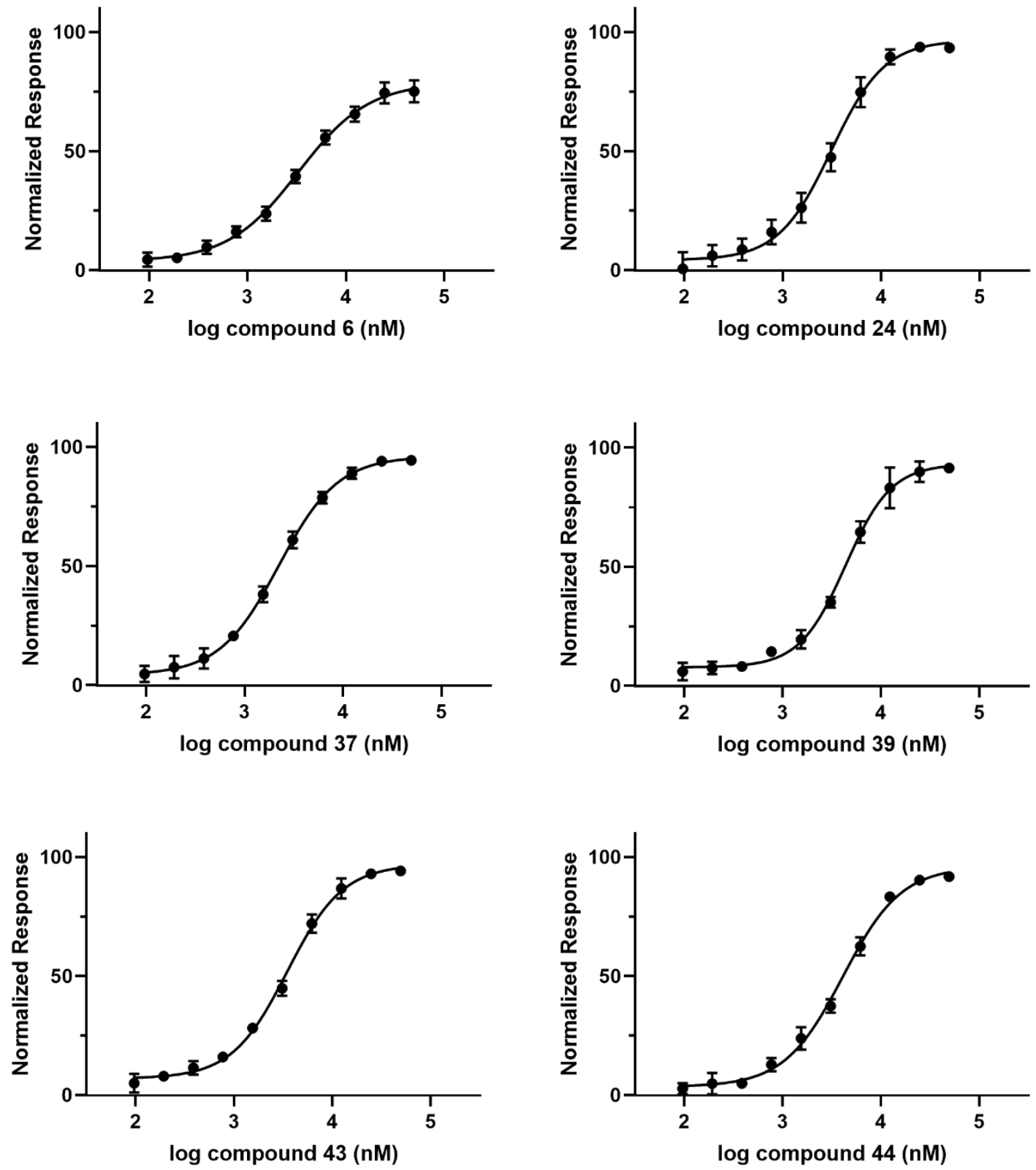

Figure S4. Dose response curves of representative compounds against MCL-1. IC 50 data represents means and SDs for four technical experiments on compounds in a 10-point dilution series against MCL-1 using the AlphaScreen assay format. ${ }^{1,2}$ 
Table S1. Diffraction data and refinement statistics. ${ }^{a}$

\begin{tabular}{|c|c|c|c|}
\hline & $\begin{array}{c}\text { BCL-XL: } \mathbf{4 3} \\
\text { (Crystal form 1) }\end{array}$ & $\begin{array}{c}\text { BCL-XL: } \mathbf{4 3} \\
\text { (Crystal form 2) } \\
\end{array}$ & sBCL-2: ABT-737 (1) \\
\hline \multicolumn{4}{|l|}{ Data collection } \\
\hline Space group & $C 222_{1}$ & $P 2_{1} 2_{1} 2_{1}$ & $P 12_{1} 1$ \\
\hline Unit cell parameters & $\begin{array}{c}66.6,112.9,204.6 \\
90,90,90\end{array}$ & $\begin{array}{c}40.3,56.3,117.8 \\
90,90,90\end{array}$ & $\begin{array}{c}100.0,67.8,100.9 \\
90,118.8,90\end{array}$ \\
\hline Resolution $(\AA)$ & $\begin{array}{l}35.71-1.30 \\
(1.33-1.30)\end{array}$ & $\begin{array}{l}38.18-2.05 \\
(2.11-2.05)\end{array}$ & $\begin{array}{l}86.47-2.75 \\
(2.85-2.75)\end{array}$ \\
\hline No. of observations & $150,754(7,622)$ & $105,560(8,064)$ & $61,109(6,035)$ \\
\hline No. of unique reflections & $35,752(1,765)$ & $17,473(1,343)$ & $30,837(3,053)$ \\
\hline Completeness (\%) & $98.2(99.6)$ & $99.7(99.8)$ & $98.95(98.4)$ \\
\hline Redundancy & $4.2(4.3)$ & $6.0(6.0)$ & $2.0(2.0)$ \\
\hline$R_{\text {merge }}(\%)$ & $2.6(66.1)$ & $7.9(42.0)$ & $8.5(116.9)$ \\
\hline$R_{\mathrm{pim}}(\%)$ & $1.7(41.1)$ & $3.8(24.4)$ & $8.5(116.9)$ \\
\hline $\mathrm{CC}_{1 / 2}$ & $0.999(0.859)$ & $0.995(0.888)$ & $0.996(0.309)$ \\
\hline Average $I / \sigma(I)$ & $14.5(2.0)$ & $14.4(2.4)$ & $6.43(0.64)$ \\
\hline \multicolumn{4}{|l|}{ Refinement } \\
\hline$R(\%)$ & $16.5(26.6)$ & $18.9(37.0)$ & $23.4(38.4)$ \\
\hline$R_{\text {free }}(\%)$ & $19.9(30.3)$ & $24.6(36.8)$ & $25.6(42.5)$ \\
\hline $\begin{array}{l}\text { No. (\%) of reflections in test } \\
\text { set }\end{array}$ & $1,824(5.1)$ & $891(5.2)$ & $1547(5.0)$ \\
\hline $\begin{array}{l}\text { No. of protein molecules per } \\
\text { asu }\end{array}$ & 1 & 2 & 6 \\
\hline R.m.s.d bond length $(\AA)$ & 0.008 & 0.006 & 0.012 \\
\hline R.m.s.d bond angle $\left(^{\circ}\right)$ & 1.45 & 1.36 & 1.91 \\
\hline Average B-factors $\left(\AA^{2}\right)^{b}$ & 21.7 & 28.3 & 78.8 \\
\hline Protein molecules & 22.5 & 30.6 & 78.2 \\
\hline Ligand & 17.9 & 24.7 & 94.7 \\
\hline Water molecules & 31.9 & 36.9 & 55.1 \\
\hline \multicolumn{4}{|l|}{ Ramachandran plot } \\
\hline \multicolumn{4}{|l|}{$\begin{array}{l}\text { Residues other than Gly and } \\
\text { Pro in: }\end{array}$} \\
\hline Most favoured regions (\%) & 99.3 & 97.5 & 99.3 \\
\hline $\begin{array}{l}\text { Additional allowed regions } \\
(\%)\end{array}$ & 0.7 & 2.5 & 0.7 \\
\hline Disallowed regions $(\%)$ & 0 & 0 & 0 \\
\hline PDB code & 7JGW & 7JGV & 7JMT \\
\hline
\end{tabular}

${ }^{a}$ Values in parentheses are for the highest-resolution shell.

${ }^{\mathrm{b}}$ Calculated by BAVERAGE in CCP4 suite. ${ }^{3}$ 
Table S2. Evaluation of compounds in a S. mansoni parasite death assay.

\begin{tabular}{|c|c|c|c|}
\hline \multirow{2}{*}{ Cmpnd } & \multicolumn{3}{|c|}{ S. mansoni adult \% viability at $\mathbf{7 2} \mathbf{h}^{a}$} \\
\cline { 2 - 4 } & $\mathbf{1 0 0} \boldsymbol{\mu M}$ & $\mathbf{5 0} \boldsymbol{\mu M}$ & $\mathbf{1 0} \boldsymbol{\mu M}$ \\
\hline $\mathbf{3 8}$ & 54.8 & 54.4 & 25.2 \\
\hline $\mathbf{4 3}$ & 31.2 & 28.9 & 21.6 \\
\hline
\end{tabular}

${ }^{a}$ Adult $S$. mansoni worms were treated with compounds at either 10,50 or $100 \mathrm{uM}$ for $72 \mathrm{~h}$ and viability of worms evaluated via microscopy. Data represents the average of duplicate experiments. 


\section{REFERENCES}

(1). Lessene, G.; Czabotar, P. E.; Sleebs, B. E.; Zobel, K.; Lowes, K. N.; Adams, J. M.; Baell, J. B.; Colman, P. M.; Deshayes, K.; Fairbrother, W. J.; Flygare, J. A.; Gibbons, P.; Kersten, W. J.; Kulasegaram, S.; Moss, R. M.; Parisot, J. P.; Smith, B. J.; Street, I. P.; Yang, H.; Huang, D. C.; Watson, K. G. Structure-guided design of a selective BCL-X(L) inhibitor. Nat. Chem. Biol. 2013, 9 (6), 390-397.

(2). Sleebs, B. E.; Kersten, W. J. A.; Kulasegaram, S.; Nikolakopoulos, G.; Hatzis, E.; Moss, R. M.; Parisot, J. P.; Yang, H.; Czabotar, P. E.; Fairlie, W. D.; Lee, E. F.; Adams, J. M.; Chen, L.; van Delft, M. F.; Lowes, K. N.; Wei, A.; Huang, D. C. S.; Colman, P. M.; Street, I. P.; Baell, J. B.; Watson, K.; Lessene, G. Discovery of potent and selective benzothiazole hydrazone inhibitors of Bcl-XL. J. Med. Chem. 2013, 56 (13), 5514-5540.

(3). Winn, M. D.; Ballard, C. C.; Cowtan, K. D.; Dodson, E. J.; Emsley, P.; Evans, P. R.; Keegan, R. M.; Krissinel, E. B.; Leslie, A. G. W.; McCoy, A.; McNicholas, S. J.; Murshudov, G. N.; Pannu, N. S.; Potterton, E. A.; Powell, H. R.; Read, R. J.; Vagin, A.; Wilson, K. S. Overview of the CCP4 suite and current developments. Acta Crystallogr. D Biol. Crystallogr. 2011, 67 (Pt 4), 235-242. 